\title{
Radial propagation of geodesic acoustic modes in up-down asymmetric magnetic geometries
}

\author{
Robert Hager* and Klaus Hallatschek ${ }^{\dagger}$ \\ Max-Planck-Institut für Plasmaphysik \\ Boltzmannstraße 2, D-85748, Garching, Germany
}

(Dated: February 19, 2010)

\begin{abstract}
The energy approach to the analysis of the propagation of geodesic acoustic modes presented in Physics of Plasmas 16, 072503 (2009) is generalized to up-down asymmetric magnetic geometries including a model for single-null configuration. By removing the neoclassical cancellation effects, updown asymmetry can trigger a non-vanishing group velocity at zero radial wavenumber. Theoretical insight in this effect is provided by analytical calculations combined with numerical gyrokinetic and two-fluid studies. Thereby, an useful estimate of the group velocity at zero radial wavenumber is derived within a two-fluid framework.
\end{abstract}

\section{INTRODUCTION}

Radial propagation of geodesic acoustic modes (GAMs) [1], and GAM eigenmodes have been discussed in recent literature [2-4]. The existence and properties of global GAM eigenmodes, which are influenced by the GAM group velocity, might be relevant for the efficiency of GAM excitation $[4,5]$. This, in turn, could affect turbulent transport due to the potential role of GAMs in nonlinear turbulence saturation [6].

In this context we have presented a method to estimate the radial group velocity of a GAM by comparing its energy flux to its total free energy [5]. The energy flux of GAMs consists of two kinds of transports, the advection of free energy with the magnetic inhomogeneity drift (curvature, $\nabla B$ ) and the polarization energy flux. In case of up-down symmetric plasma equilibria, which have been used in Ref. [5], the magnetic inhomogeneity fluxes are of order $k_{r} \rho_{s e} v_{d} E_{f l u c}$ due to neoclassical cancellation effects, where $v_{d}$ is the sum of curvature and $\nabla B$ drifts and $E_{f l u c}$ is the energy of the fluctuations. Since the polarization fluxes are also of order $k_{r} \rho_{s e} v_{d} E_{f l u c}$ and opposite to the curvature energy-flux, the observed group velocities are much smaller than $v_{d}$. Here as well as in the following, $r$ is defined as the minor radius of a particular flux surface at the outboard midplane, which makes $r$ a flux surface label. Thus, $k_{r}$ is the wavenumber and $v_{r}$ the velocity with respect to the coordinate $r$.

In this article, we extend the analysis presented in Ref. [5] to up-down asymmetric magnetic geometries including a model for single-null configuration. In these cases, the curvature energy-fluxes can be of order $v_{d} E_{\text {fluc }}$ since the asymmetry removes the neoclassical cancellation, and larger group velocities of order $v_{d}$ and above are expected.

\footnotetext{
*Electronic address: robert.hager@ipp.mpg.de

$\dagger$ Electronic address: klaus.hallatschek@ipp.mpg.de
}

\section{FLUID MODEL}

The units are chosen such that the magnetic drift velocity equals unity at the outboard midplane. Density $n$, ion and electron temperature $T_{i}$ and $T_{e}$, and electric potential perturbations $\phi$ are normalized to $\rho^{\star} n_{0}, \rho^{\star} T_{0, i / e}$, $\rho^{\star} T_{0, e} / e$, respectively, where the subscript 0 indicates the corresponding background value and $\rho^{\star}$ is given by $\rho_{s e} / R_{0}$ with the major torus radius at the outboard midplane $R_{0}, c_{s e} \equiv\left(T_{0, e} / m_{i}\right)^{1 / 2}$, and $\rho_{s e} \equiv\left(m_{i} c_{s e}\right) /\left(e B_{0}\right)$. The time scale is $t_{0} \equiv R_{0} /\left(2 c_{s e}\right)$.

The evaluation of the time derivative of the total free energy for the cold ion equations in Ref. [5] in general geometry yields:

$$
\begin{aligned}
\partial_{t}\langle E\rangle & =\left\langle-\nabla \cdot\left(\frac{\boldsymbol{v}_{d} n^{2}}{2}\right)+\nabla \cdot \frac{(n \nabla \dot{n})}{B_{r e l}^{2}}\right. \\
& \left.+\nabla \cdot \frac{\left(n \nabla \dot{\phi}_{0}\right)}{B_{r e l}^{2}}\right\rangle
\end{aligned}
$$

where $\boldsymbol{v}_{d}$ is the sum of curvature and $\nabla B$-drift, and $B_{\text {rel }} \equiv B / B_{0}$. The additional factor $1 / B_{\text {rel }}$ appears because the polarization related terms in [5] implicitly contain a factor $\rho_{i}^{2}$ (normalized to its value at the outboard midplane). The flux-surface average in general geometry is defined by $A_{0} \equiv\langle A\rangle \equiv\left(\oint B^{-1} \mathrm{~d} l_{\|}\right)^{-1} \oint A B^{-1} \mathrm{~d} l_{\|}$, where $\mathrm{d} l_{\|}$denotes the line element parallel to the magnetic field. With adiabatic electrons, $\phi=\phi_{0}+n$ and $n_{0}=0$ still hold.

The first term on the right hand side of Eq. (1) is the flow of the energy of the electron pressure perturbations in the ion magnetic drift direction. The second term is a polarization energy-flux, which can be interpreted as an hydraulic energy-flux, $p_{e} \boldsymbol{v}_{\text {pol }}$, consisting of the electron pressure and the polarization drift velocity. A detailed discussion of the two terms can be found in Ref. [5]. The term $n \nabla \dot{\phi}_{0} / B_{\text {rel }}^{2}$ appearing in Eq. (1) is an additional component of the polarization energy-flux caused by the flux-surface averaged potential $\phi_{0}$, and vanishes for updown-symmetric magnetic geometries.

In [5] the density perturbation for $k_{r} \ll 1$ was approx- 
imated by

$$
n^{2} \approx \frac{4 v_{E}^{2}}{\omega^{2} R^{2}}\left(1+\frac{2 v_{d, r}}{v_{p}}\right)
$$

with the $E \times B$-drift velocity $v_{E}$, the radial component of the magnetic inhomogeneity drift $v_{d, r}$, the major radius $R$ and the GAM phase velocity $v_{p}$. Whereas in circular geometry, the first term in Eq. (2) and the last term on the right hand side of Eq. (1) do not contribute to the flux-surface averaged energy flux - implying zero group velocity for $k_{r}=0$ - in general geometries, they can give rise to a non-vanishing $v_{g}\left(k_{r}=0\right)$ and are much larger than the $k_{r}$-dependent part of the polarization energyflux, $n \nabla \dot{n} / B_{\text {rel }}^{2}$.

Circular flux-surfaces [7] augmented with an rdependent vertical shift $Z_{0}(r)$, i.e. $R(r, \theta)=R_{0}+r \cos (\theta)$ and $Z(r, \theta)=Z_{0}(r)-r \sin (\theta)$, may serve as the most straightforward test of up-down asymmetric geometry in numerical studies. The Z-shifted geometry is the simplest modification of the circular equilibrium which shows the basic effects of up-down asymmetry while avoiding the complexity of force-free asymmetric configurations. Lacking complete consistency, it can be thought of as being maintained by a conductor inside the considered flux surface. We have studied the dependence of $v_{g}\left(k_{r}=0\right)$ on the differential Z-shift $s_{z} \equiv \partial_{r} Z_{0}$ (which can take values between -1 and 1 ) for cold ions and infinite safety factor using the two fluid code NLET [8], the gyrokinetic codes GS2 [9] and GYRO [10], and direct numerical solutions of the GAM equation.

A GAM spectrum computed with NLET, in which $v_{d}$ is parallel to the Z-axis, for $s_{z}=0.3$ is shown in Fig. 1. As an effect of the additional $k_{r}$-independent terms in the group velocity, the extremum of the GAM dispersion is shifted away from $k_{r}=0$. The group velocity at $k_{r}=0$ is positive. Thus, as conjectured in Ref. [5], $v_{g}\left(k_{r}=0\right) \neq 0$ due to the up-down asymmetry of the flux-surfaces.

The group velocity at $k_{r}=0$ is rather accurately linear in $\left(1+\left|s_{z}\right|\right) /\left(1-\left|s_{z}\right|\right)$ (Fig. 2), the ratio of the inverse fluxsurface distances at the poloidal positions of the maximum and the minimum of the poloidal magnetic field, i.e. $|\nabla r(\theta=\pi / 2)| /|\nabla r(\theta=-\pi / 2)|$ for $s_{z}>0$. This implies $v_{g}\left(k_{r}=0\right) \rightarrow \infty$ for $s_{z} \rightarrow 1$.

A very realistic up-down asymmetric magnetic geometry can be constructed with the magnetic field of three toroidal current loops, representing the plasma current and the currents of two coils, used to elongate the plasma and to generate separatrices. For large aspect ratios, the corresponding vector potentials are given by $A_{i}=a_{i} / 2 \ln \left(\left(R_{0}+R\right)^{2}+\left(Z+z_{i}\right)^{2}\right)$ where $a_{i}$ measures the current and $z_{i}$ the position of conductor $i$ on the $Z$ axis. Since, due to axisymmetry, equipotential surfaces of the vector potential (of the poloidal field) are also surfaces of constant poloidal flux, the flux-surface shape is determined by the condition $\sum_{i=0}^{2} A_{i}=\Psi=$ const. with $\Psi_{\max } \equiv \Psi$ for the last closed flux-surface. In order to assure that the vertical forces on the flux surface balance to zero, we choose $a_{1}=m a_{2}$ and $z_{1}=-m z_{2}$ with

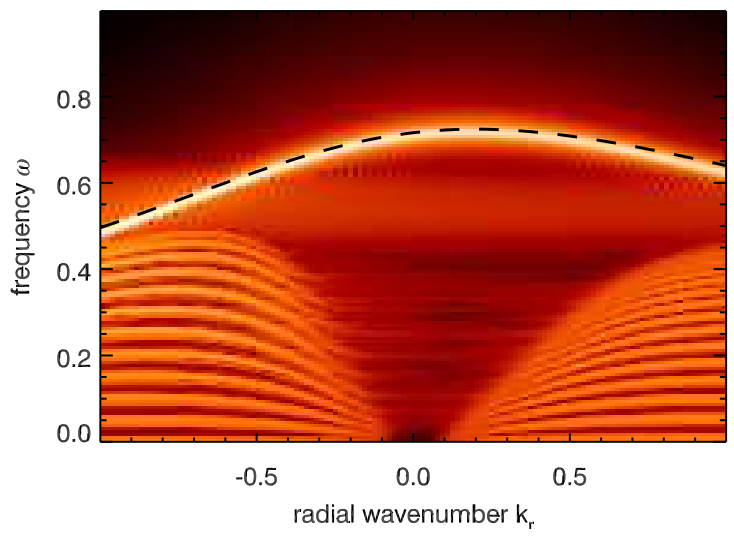

FIG. 1: (color online) NLET computed log-color-coded GAM spectrum (Fourier transform of $\phi$ ) for $\tau=0, q=100$, and $s_{z}=0.3$ with direct numerical solution of the GAM equations (dashed). The extremum of the GAM dispersion is shifted along the $k_{r}$-axis due to the up-down asymmetric magnetic geometry.

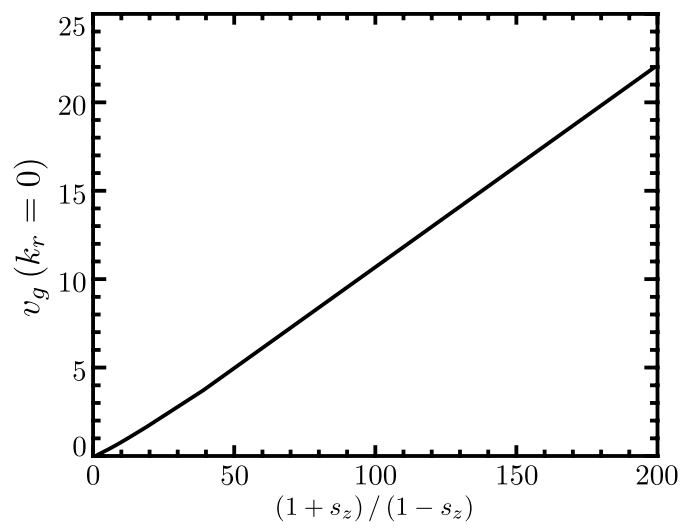

FIG. 2: GAM group velocity $v_{g}\left(k_{r}=0\right)$ in shifted Miller geometry plotted against $\left(1+s_{z}\right) /\left(1-s_{z}\right)$. For $s_{z} \rightarrow 1$, the ratio $\left(1+s_{z}\right) /\left(1-s_{z}\right)$ diverges, implying $v_{g}\left(k_{r}=0\right) \rightarrow \infty$.

a real factor $m>0$. The necessary geometry data can then be calculated analogous to an ordinary Miller equilibrium [7]. As in Miller geometry we define $r$ to be the minor radius of a particular flux surface at the outboard midplane. The corresponding value at the separatrix is referred to as $r_{\max }$.

In this geometry, $\left(1+\left|s_{z}\right|\right) /\left(1-\left|s_{z}\right|\right)$ corresponds to $x \equiv|\nabla r(\theta=-\pi / 2)| /|\nabla r(\theta=\pi / 2)|$. Thus, the separatrix geometry can be compared to the shifted circular geometry by plotting $v_{g}$ versus $s_{z} \equiv(x-1) /(x+1)$ (Fig. $3)$. The group velocity at $k_{r}=0$ obtained from the numerical solution of the GAM equations for $a_{0}=1$, $z_{0}=0, a_{1}=2 a_{2}=2, z_{1}=-2 z_{2}=2$ is positive. The values of $s_{z}$ for which $v_{g}$ is plotted correspond to $r=(0.055 \ldots 0.999) r_{\max }$. For small $s_{z}$, the group velocities of the shifted circular and the separatrix geometry are of the same order, but for $s_{z} \rightarrow 1$ the latter stays 


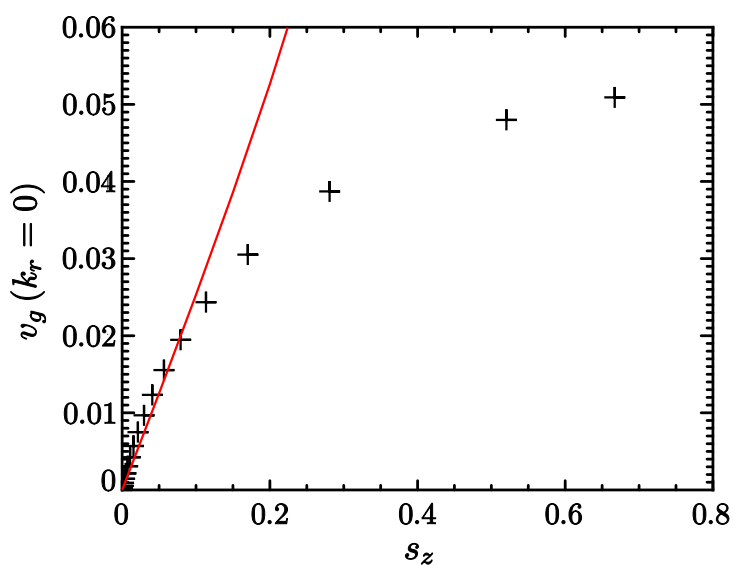

FIG. 3: (color online) GAM group velocity $v_{g}\left(k_{r}=0\right)$ in single-null (crosses) and shifted Miller geometry (solid) plotted against $s_{z}$. When approaching the X-point, i.e. $s_{z} \rightarrow 1$, the single-null group velocity approaches a finite maximum, while $v_{g}$ diverges in case of the Miller equilibrium with $Z$ shift.

finite whereas the former diverges.

An estimate of the group velocity for $\tau>0$ and large safety factor can be derived by evaluating the two-fluid Poynting flux for warm ions and infinite safety factor. This in turn is obtained by a straightforward modification of Eq. (15) of Ref. [5] to general geometry - analogous to Eq. (1) - and adding the third term on the right hand side of Eq. (1). In the infinite safety factor limit, we can neglect all terms involving $\boldsymbol{v}_{\|} \cdot \nabla$, and since $k_{\theta} \ll k_{r}$ for GAMs, we only have to consider the radial component of $\nabla_{\perp}$. In flux coordinates using the normalization defined at the beginning of this section, we can write $\nabla_{\perp}=\gamma \partial_{\Psi} \equiv \gamma_{r e l} \partial_{r}$. Here, $\Psi$ is the poloidal flux, $\gamma \equiv|\nabla \Psi|, \gamma_{0}$ is the value of $\gamma$ at the outboard midplane, and $\gamma_{r e l} \equiv \gamma / \gamma_{0}$. The partial derivative $\partial_{r}$ corresponds to $i k_{r}$ in Fourier space, where $k_{r}$ is the minor radial wave number at the outboard midplane. Thus, the radial component of the curvature operator $\hat{C} \equiv-\boldsymbol{v}_{d} \cdot \nabla$ is $-v_{d, r} \partial_{r}$ with

$$
v_{d, r} \equiv \gamma_{r e l} \boldsymbol{v}_{d} \cdot \frac{\nabla \Psi}{|\nabla \Psi|}=\frac{R_{0} \gamma_{r e l}}{B_{r e l}}(\hat{\boldsymbol{b}} \times \nabla \ln B) \cdot \frac{\nabla \Psi}{|\nabla \Psi|} .
$$

We use the ordering $k_{r} \phi_{0} \sim n \sim T_{i} \sim E_{\text {fluc }}^{1 / 2}$ and keep only Poynting fluxes of order $v_{d} E_{\text {fluc }}$. Assuming constant ion entropy, $T_{i} \approx 2 n / 3$, and neglecting polarization effects, $n \approx\left(v_{d, r} k_{r} \phi_{0}\right) / \omega$, where $\omega \approx \omega\left(k_{r}=0\right) \approx$ $((1+5 \tau / 3) / 2)^{1 / 2}$. The total free energy of the GAM is $E \approx k_{r}^{2} \phi_{0}^{2}\left\langle\gamma_{\text {rel }}^{2} / B_{\text {rel }}^{2}\right\rangle$. Combining everything, one obtains the group velocity

$$
\begin{aligned}
v_{g}(\tau) & \approx \frac{\left(1+\frac{5 \tau}{3}\right)^{2}+\frac{10 \tau^{2}}{9}}{\left(1+\frac{5 \tau}{3}\right)}\left\langle v_{d, r}^{3}\right\rangle_{g}+\frac{11 \tau}{12}\left\langle\frac{\gamma_{r e l}^{2} v_{d, r}}{B_{r e l}^{2}}\right\rangle_{g} \\
& -\left(1+\frac{5 \tau}{3}\right)\left\langle\frac{\gamma_{r e l}^{2} v_{d, r}}{B_{r e l}^{2}}\right\rangle_{g},
\end{aligned}
$$

where $\langle\ldots\rangle_{g} \equiv\left\langle\gamma_{\text {rel }}^{2} / B_{\text {rel }}^{2}\right\rangle^{-1}\langle\ldots\rangle$. The first term on the right hand side of Eq. (4) corresponds to the first term in Eq. (1), the second term is a finite Larmor radius (FLR) correction to the first one. The third term corresponds to the polarization energy-flux $n \nabla \dot{\phi}_{0} / B_{\text {rel }}^{2}$ in Eq. (1).

With large aspect ratio and small $s_{z}, v_{d, r} \approx$ $-\sin (\theta)\left(1+s_{z} \sin (\theta)\right)$ and $\gamma_{r e l} \approx\left(1-s_{z} \sin (\theta)\right)^{-1}$. Thus, to first order in $s_{z}$ one obtains $\left\langle v_{d, r}^{3}\right\rangle_{g} \approx-3 s_{z} / 4$ and $\left\langle\gamma_{r e l}^{2} v_{d, r} / B_{r e l}^{2}\right\rangle_{g} \approx-s_{z}$.

As mentioned before, the parameters $a_{i}$ and $z_{i}$ of the separatrix geometry have been chosen such that the vertical magnetic forces on the central current loop (i.e. the flux surfaces) balance, which corresponds to the condition $F_{Z}=\iint p_{\perp} \hat{\rho}_{Z} \mathrm{~d} f=0$ on each flux surface. Here, $p_{\perp}=B^{2} /\left(2 \mu_{0}\right)$ is the perpendicular component of the magnetic pressure, $\hat{\rho}_{Z}$ the vertical component of the fluxsurface normal vector, and $\mathrm{d} f$ is an infinitesimal fluxsurface element. This can be reduced to $\oint \gamma^{2} \partial_{\|} \ln R \mathrm{~d} l_{\|}=$ 0 . By expressing $v_{d, r}=F B_{0} R_{0} / \gamma_{0}\left(\partial_{\|} \ln B\right) / B$, one obtains

$$
\left\langle\frac{\gamma_{r e l}^{2} v_{d, r}}{B_{r e l}^{2}}\right\rangle=\frac{1}{\oint B^{-1} \mathrm{~d} l_{\|}} \frac{F B_{0}^{3} R_{0}}{\gamma_{0}^{3}} \oint \frac{\gamma^{2}}{B^{4}} \partial_{\|} \ln B \mathrm{~d} l_{\|}
$$

For large aspect ratio, $B$ is approximately constant and, due to $q \gg 1, \partial_{\|} \ln B \approx-\partial_{\|} \ln R$. Thus, the second integral on the right hand side of Eq. (5) reduces to the force-balance condition.

Therefore, $\left\langle\gamma_{r e l}^{2} v_{d, r} / B_{r e l}^{2}\right\rangle_{g} \approx 0$ in contrast to the shifted-circle geometry. This implies that for $k_{r}=0$, the energy of GAMs is transported essentially by the magnetic drift energy-flux $v_{d} E_{f l u c}$. In case of vertical forcebalance this result agrees with the conjecture in Ref. [5], that in single-null geometry $v_{g}\left(k_{r}=0\right)$ has the sign of $v_{d, r}$ at the position opposite to the $\mathrm{X}$-point. However, with low aspect ratio, when the variation of $B$ across the flux-surface cannot be neglected any longer, the remaining two terms in Eq. (4) might be significant.

Numerical calculations suggest that for large aspect ratio one can approximate the geometry coefficient $\left\langle v_{d, r}^{3}\right\rangle_{g}$ by $0.028\left(1-\left(s_{z}-1\right)^{4}\right)^{-1 / 2}$ for $a_{0}=1, z_{0}=0, a_{1}=2 a_{2}=$ 2 , and $z_{1}=-2 z_{2}=2$.

\section{GYROKINETIC MODEL}

To elaborate the analogies between gyrokinetic and two-fluid theory, we return to the linear gyrokinetic framework already used in Ref. [5]. As in our previous article, we only treat cases, in which the parallel ion dynamics can be neglected, i.e. high safety factors $q$. In practice, this is hardly a restriction because modes lose the character of a GAM due to resonances with soundwaves at low $q[5,11]$. For effects of finite orbit width (FOW) on GAMs in that regime see e.g. Ref. [12].

With arbitrary geometry but infinite safety factor, the 
expression for $\partial_{t}\langle E\rangle$ is given by:

$$
\begin{aligned}
\partial_{t}\langle E\rangle & =\left\langle-\int\left[\nabla \cdot \frac{\boldsymbol{v}_{d}}{\tau} \frac{K^{2}}{2 F_{0}}-\left\{\frac{\boldsymbol{v}_{d}}{\tau} \cdot \nabla K, n\right\}_{\hat{J}_{0}}\right.\right. \\
& \left.+\left\{\tau f, \frac{\boldsymbol{v}_{d}}{\tau} \cdot \nabla \phi_{0}\right\}_{\hat{J}_{0}}+\left\{n F_{0}, \frac{\boldsymbol{v}_{d}}{\tau} \cdot \nabla \phi_{0}\right\}_{\hat{J}_{0}^{2}}\right] \mathrm{d}^{3} v \\
& \left.-\frac{1}{2}\{\phi, \dot{\phi}\}_{\frac{1-\hat{\Gamma}_{0}}{\tau}}+\nabla \cdot\left(\phi_{0} \hat{\chi} \partial_{t} \boldsymbol{E}\right)\right\rangle
\end{aligned}
$$

The velocity $\boldsymbol{v}_{\mathrm{d}}$ is the sum of the curvature and $\nabla B$ drifts of the individual particles. $F_{0}$ is the thermal background distribution function, which is normalized such that $\int F_{0} \mathrm{~d}^{3} v=1$. Gyro-averaging is represented by the operator $\hat{J}_{0}$, the thermal average of $\hat{J}_{0}^{2}$ is defined by $\hat{\Gamma}_{0} \equiv \int F_{0} \hat{J}_{0}^{2} \mathrm{~d}^{3} v, K \equiv \tau f+\hat{J}_{0} n F_{0}$ and $\hat{\chi}$ is the susceptibility operator. The brackets denote $\{a, b\}_{K} \equiv$ $a(K * b)-b(K * a)$, with $*$ indicating convolutions. $\{a, b\}_{K}$ can always be written as a divergence, provided the kernel $K$ is symmetric [5].

As in section II, one can write the curvature operator $\boldsymbol{v}_{d} \cdot \nabla$ as $v_{d, r} \partial_{r}$ with the appropriate kinetic expression for $v_{d, r}$ when evaluating Eq. (6). The operators $\hat{J}_{0}, \hat{\Gamma}_{0}$ and $\hat{\chi}$ are defined by their Fourier transforms (using flux coordinates and the normalization given in Sec. II). The gyro-average $\hat{J}_{0}$ corresponds to $J_{0}\left(\tau^{1 / 2} v_{\perp} k_{r} \gamma_{r e l} / B_{r e l}\right)$, the thermal average of $\hat{J}_{0}^{2}, \hat{\Gamma}_{0}$, to $\Gamma_{0}\left(k_{r}\right) \equiv \exp \left(-\tau k_{r}^{2} \gamma_{\text {rel }}^{2} / B_{\text {rel }}^{2}\right) I_{0}\left(\tau k_{r}^{2} \gamma_{\text {rel }}^{2} / B_{\text {rel }}^{2}\right)$, and the susceptibility operator $\hat{\chi}$ to $\chi\left(k_{r}\right) \equiv\left(\gamma_{\text {rel }}^{2} / B_{\text {rel }}^{2}\right)(1-$ $\left.\Gamma_{0}\right) /\left(\tau k_{r}^{2}\right)$ with the Bessel function of the first kind $J_{0}$ and the modified Bessel function of the first kind $I_{0}$.

The first term on the right hand side of Eq. (6) represents the advection of the free energy of gyro-averaged fluctuations by magnetic drifts. It is the equivalent to the first term on the right hand side of Eq. (1). Since $k_{r} \phi_{0} \sim n \sim E_{\text {fluc }}^{1 / 2}$, this energy flux is of order $v_{d} E_{\text {fluc }}$. The remaining three terms in the integral are FLR corrections. As $J_{0}$ is $O\left(k_{r}^{2}\right)$ in the commutator brackets, those terms correspond to energy fluxes of order $k_{r} \rho_{s e} v_{d} E_{f l u c}$ and will therefore be neglected. The last two terms describe the polarization energy-flux.

Different from Eq. (20) in Ref. [5], the very last term contains the entire electric field instead of its flux-surface average. Analogous to Ref. [5] the polarization energyflux can be expressed as

$$
-\frac{\gamma_{r e l}^{2} \chi}{B_{r e l}^{2}}\left[k_{r} \omega\left(\phi_{0} n+n^{2}\right)+v_{p} \frac{\chi\left|k_{r} \phi\right|^{2}}{2} \frac{\partial \ln \chi}{\partial \ln k_{r}}\right] .
$$

Since $\chi$ is $O(1)$ and $v_{p} \chi\left(\partial \ln \chi / \partial \ln k_{r}\right)=\omega\left(\partial \chi / \partial k_{r}\right)$ is $O\left(k_{r}\right)$, only the very first term in Eq. (7) contributes to $v_{g}\left(k_{r}=0\right)$. In Ref. [5] this term vanished due to fluxsurface symmetry. It can therefore be considered as the equivalent of the additional polarization flux in Eq. (1).

We have studied the $\tau$-dependence of $v_{g}\left(k_{r}=0\right)$ for the shifted circular flux-surfaces defined in section II using the gyrokinetic codes GS2 [9] and GYRO [10]. The plot of $v_{g}\left(k_{r}=0\right)$ for $s_{z}=0.3$ and 0.5 against $\tau$ (Fig.

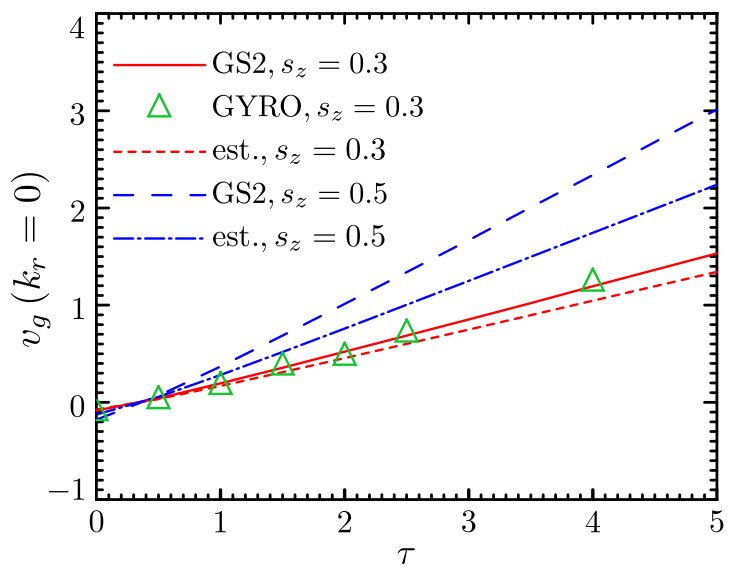

FIG. 4: (color online) GAM group velocity - computed with GS2, GYRO and with the estimate (4) - at $k_{r}=0$ for $s_{z}=0.3$ and $s_{z}=0.5$ vs. the ratio of ion to electron temperature $\tau$. Since contrary to NLET, GS2 and GYRO take $v_{d}$ to be in negative Z-direction, one has to compare the results with Eq. (4) for $s_{z} \rightarrow-s_{z}$.

4) illustrates that the additional polarization flux discussed here [see Eq. (7)] and in section II exceeds the energy flux due to the magnetic inhomogeneity drift in the cold ion case. When the ion temperature is increased $(\tau \gtrsim 0.3)$ gyroradius effects overcompensate this effect such that the group velocity changes sign and behaves as suggested in Ref. [5]. This behavior and the order of magnitude of $v_{g}$ is quite well reproduced by our two-fluid approximation (4).

\section{CONCLUSIONS}

We have studied the effects of up-down asymmetric magnetic geometry on the propagation of geodesic acoustic modes.

The two-fluid expression for the Poynting flux of GAMs in the cold ion and infinite safety factor limit with large aspect ratio circular flux-surfaces derived in Ref. [5] has been generalized to arbitrary toroidal geometries, yielding an additional component of the polarization energy-flux of order $v_{d} E_{f l u c}$. Together with the curvature energy-flux of the same order - caused by the up-down asymmetry eliminating neoclassical cancellation effects it leads to a non-vanishing group velocity at $k_{r}=0$, the sign and magnitude of which depend on the ratio of ion to electron temperature, $\tau$, and the magnetic geometry. Analogous terms have been found in the generalized gyrokinetic expression of the GAM Poynting flux.

The geometry and temperature dependences of $v_{g}\left(k_{r}=0\right)$ have been studied numerically using the twofluid code NLET, and the gyrokinetic codes GS2 and GYRO as well as analytically by deriving a group velocity estimate [see Eq. (4)] for arbitrary $\tau$ in a two-fluid framework. By means of the estimate, we have found that it is an important for the energy transport at large aspect 
ratio, whether the vertical magnetic forces on the fluxsurfaces balance or not. While in the former case, the energy of GAMs is transported essentially by the magnetic inhomogeneity drift - which confirms the conjecture in Ref. [5], that in single-null geometry $v_{g}\left(k_{r}=0\right)$ has the sign of $v_{d, r}$ at the position opposite to the X-point the polarization energy-flux might become important in the latter case.

In order to have high GAM group velocity, the magnetic geometry should have a maximum of the poloidal magnetic field - corresponding to the flux-surfaces being close to each other - at a poloidal position where the magnetic inhomogeneity drift has a significant radial component. In case of the single-null geometry in Sec. II, this could be realized by adding an indentation coil - with a current opposite to the elongation currents - close to the plasma column opposite to the X-point. Preliminary studies easily yield a group velocity three times larger that way. In a real device, such a configuration could be generated by appropriately combining the plasma shaping coils, for example in DIII-D, where the shaping system is located close to the plasma [13]. Altering the direction and speed of the GAM propagation might be used to search for a potential influence on the confinement and even on the H-mode. For example, it would be interesting to determine whether the dependence of the $\mathrm{H}$-mode-power-threshold on the magnetic drift direction is really due to its relation to the $\mathrm{X}$-point or possibly rather due to its influence on the GAM propagation direction.
[1] N. Winsor, J. L. Johnson, and J. M. Dawson, Physics of Fluids 11, 2448 (1968).

[2] F. Zonca and L. Chen, Europhysics Letters 83, 35001 (2008).

[3] X. Q. Xu, Z. Xiong, Z. Gao, W. M. Nevins, and G. R. McKee, Phys. Rev. Lett. 100, 215001 (2008).

[4] K. Itoh, S.-I. Itoh, P. H. Diamond, A. Fujisawa, M. Yagi, T. Watari, Y. Nagashima, and A. Fukuyama, Plasma Fusion Res. 1, 037 (2006).

[5] R. Hager and K. Hallatschek, Physics of Plasmas 16, 072503 (2009).

[6] R. E. Waltz and C. Holland, Physics of Plasmas 15, 122503 (2008).

[7] R. L. Miller, M. S. Chu, J. M. Greene, Y. R. Lin-Liu, and R. E. Waltz, Physics of Plasmas 5, 973 (1998).

[8] K. Hallatschek and A. Zeiler, Physics of Plasmas 7, 2554 (2000).

[9] W. Dorland, F. Jenko, M. Kotschenreuther, and B. N. Rogers, Physical Review Letters 85, 5579 (2000).

[10] J. Candy and R. E. Waltz, Journal of Computational Physics 186, 545 (2003).

[11] K. Hallatschek, Plasma Physics and Controlled Fusion 49, B137 (2007).

[12] H. Sugama and T.-H. Watanabe, Physics of Plasmas 13, 012501 (2006).

[13] J. Luxon, Nuclear Fusion 42, 614 (2002). 